\title{
BMJ Global Health Chronic kidney disease in low-income to middle-income countries: the case for increased screening
}

\author{
Cindy George, ${ }^{1}$ Amelie Mogueo, ${ }^{2}$ Ikechi Okpechi, ${ }^{3,4}$ Justin B Echouffo-Tcheugui, ${ }^{5}$ \\ Andre Pascal Kengne ${ }^{1}$
}

To cite: George C, Mogueo A, Okpechi I, et al. Chronic kidney disease in low-income to middle-income countries: the case for increased screening. BMJ Glob Health 2017;2:e000256. doi:10.1136/ bmjgh-2016-000256

Received 28 November 2016 Accepted 28 March 2017

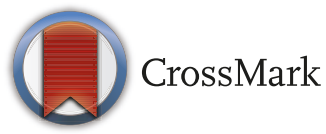

${ }^{1}$ Non-Communicable Disease Research Unit, South African Medical Research Council, Parow, Cape Town, South Africa ${ }^{2}$ Department of Management, Assessment and Health Policy, School of Public Health, The University of Montreal, Montreal, Canada

${ }^{3}$ Division of Nephrology and Hypertension, Groote Schuur Hospital and University of Cape Town, Cape Town, South Africa ${ }^{4}$ Kidney and Hypertension Research Unit, University of Cape Town, Cape Town, South Africa

${ }^{5}$ Department of Medicine Brigham and Women's Hospital, Boston, Massachusetts, USA

Correspondence to

Professor Andre Pascal Kengne andre.kengne@mrc.ac.za

\section{ABSTRACT}

Chronic kidney disease (CKD) is fast becoming a major public health issue, disproportionately burdening lowincome to middle-income countries, where detection rates remain low. We critically assessed the extant literature on CKD screening in low-income to middleincome countries. We performed a PubMed search, up to September 2016, for studies on CKD screening in low-income to middle-income countries. Relevant studies were summarised through key questions derived from the Wilson and Jungner criteria. We found that low-income to middle-income countries are ill-equipped to deal with the devastating consequences of CKD, particularly the late stages of the disease. There are acceptable and relatively simple tools that can aid CKD screening in these countries. Screening should primarily include high-risk individuals (those with hypertension, type 2 diabetes, HIV infection or aged $>60$ years), but also extend to those with suboptimal levels of risk (eg, prediabetes and prehypertension).

Since screening for hypertension, type 2 diabetes and HIV infection is already included in clinical practice guidelines in resource-poor settings, it is conceivable to couple this with simple CKD screening tests. Effective implementation of CKD screening remains a challenge, and the costeffectiveness of such an undertaking largely remains to be explored. In conclusion, for many compelling reasons, screening for CKD should be a policy priority in low-income to middle-income countries, as early intervention is likely to be effective in reducing the high burden of morbidity and mortality from CKD. This will help health systems to achieve cost-effective prevention.

\section{INTRODUCTION}

Chronic kidney disease (CKD) is an increasing global public health issue, ${ }^{1}$ with an estimated overall prevalence of $8 \%-16 \% .^{2}$ This corresponds to nearly 500 million affected individuals, of whom $78 \%$ (387.5 million) reside in low-income to middle-income countries (LMICs). ${ }^{3}$ Between 1990 and 2010, deaths attributable to CKD nearly doubled, and was ranked the 18th leading cause of death in 2012. ${ }^{4}$ Although the exact burden of CKD in LMICs has yet to be fully elucidated, it is estimated that the incidence rates might be up to four times higher than those observed

\section{Key questions}

What is already known about this topic?

- Chronic kidney disease (CKD) is increasingly common in low-income to middle-income countries due to the significant increase in non-infectious disease (particularly type 2 diabetes and hypertension) and infectious conditions such as HIV, combined with illequipped healthcare systems.

- As kidney disease progresses, it may lead to kidney failure (end-stage renal disease (ESRD)), and since most people in low-income to middle-income countries have no health insurance and limited access to healthcare, diagnosis of ESRD is thus essentially a death sentence.

- Early detection of CKD is essential because therapeutic interventions are likely to be effective if implemented early in the course of the disease.

\section{What are the new findings?}

- This review critically evaluated the available research evidence to support CKD screening in low-income to middle-income countries through a series of key questions derived from the Wilson and Jungner criteria for disease screening, and concluded that screening for CKD should be a policy priority in these resource-poor settings, as early interventions are likely to be effective in reducing the high burden of CKD morbidity and mortality.

\section{Recommendations for policy}

- Designing policies for CKD screening offers a unique opportunity to scale up CKD care and by so doing strengthen the healthcare system of low-income to middle-income countries, and thus potentially limiting future healthcare costs.

in developed countries. ${ }^{5}{ }^{6}$ In addition, in sub-Saharan Africa, CKD more commonly affects individuals aged between 20 and 50 years ${ }^{78}$ and the age of onset of end-stage renal disease (ESRD) is 20 years earlier in populations of African descent compared with other ethnic groups in Western countries (45 years vs 63 years)..$^{9}$ These statistics highlight the need for early and accurate detection of CKD 
Table 1 Wilson and Jungner criteria for disease screening

1 The condition sought should be an important health problem.

2 There should be an accepted treatment for patients with recognised disease.

3 Facilities for diagnosis and treatment should be available.

$\begin{array}{ll}4 & \text { There should be a latent or early symptomatic stage. } \\ 5 & \text { There should be a suitable test or examination. } \\ 6 & \text { The test should be acceptable to the population. } \\ 7 & \begin{array}{l}\text { The natural history of the condition, including } \\ \text { development from latent to declared disease, should be } \\ \text { adequately understood. }\end{array}\end{array}$

8 There should be an agreed policy on who to treat as patients.

9 The cost of case finding (including diagnosis and treatment of patients diagnosed) should be economically balanced in relation to possible expenditure on medical care as a whole.

10 Case finding should be a continuing process and not a 'once and for all' project.

Adapted from the $\mathrm{WHO}{ }^{15}$

in LMICS, as well as establish the mechanisms for appropriate management for screen-detected individuals.

The global increase in the incidence and prevalence of CKD is mainly driven by the rise in the prevalence of type 2 diabetes mellitus (T2D), hypertension, obesity and ageing, ${ }^{10}$ but also by the high prevalence of infectious diseases, especially HIV in sub-Saharan Africa. ${ }^{11} 12$ ESRD requires renal replacement therapy (RRT) or kidney transplant, both of which are costly to the individual and the society, imposing a major burden on health systems. Currently, about 2.6 million individuals worldwide are estimated to be receiving RRT, with $93 \%$ of these individuals residing in high-income or high-middle-income countries. ${ }^{13}$ Awareness of CKD remains limited in most LMICs, where a large proportion of individuals with CKD go undetected. ${ }^{14}$ There is a need for early detection of CKD in LMICs in order to reduce the related morbidity, mortality and economic costs. Studies suggest that early detection and treatment of CKD is feasible in low-resource settings ${ }^{2}$; however, recommendations on CKD screening are largely based on data from developed nations and may not necessarily apply to LMICs.

The main purpose of this review is to critically evaluate the available research evidence to support CKD screening in LMICs through a series of key questions derived from the Wilson and Jungner criteria for disease screening ${ }^{15}$ (table 1), rather than it being a traditional systematic review.

We searched Medline via PubMed for observational studies, randomised controlled trials or reviews pertaining to CKD screening among adults in LMICs, using relevant key terms like 'screening', 'chronic kidney (or renal) disease', 'kidney (or renal) dysfunction', 'decreased kidney (renal) function', 'end-stage renal disease', 'glomerular filtration rate', 'albuminuria', 'developing countries', 'low income countries', 'low-tomiddle income countries', 'Cockcroft-Gault equation', 'Modification of Diet in Renal Disease equation, 'CKD Epidemiology Collaboration equation', 'prevalence', 'incidence', 'type 2 diabetes', 'hypertension', 'HIV/ AIDS' and 'cardiovascular disease'. Additional searches included handsearch of reference list of relevant articles, and search of websites including those of the WHO and major global and regional professional organisations in the area of nephrology and cardiometabolic diseases. Identified entries were evaluated, irrespective of date or country of publication, to retain the most relevant in the judgement of the authors for the current review.

\section{WHAT ARE THE ARGUMENTS FOR CKD SCREENING IN LMICS? The burden of CKD}

A recent study on CKD, which included 12 LMICs, reported a CKD prevalence of $14.3 \%$ in the general population and $36.1 \%$ in the high-risk population. ${ }^{16}$ These findings are consistent with those of a previous meta-analysis of 21 studies in LMICs, in which 13.9\% of the general population had CKD, ${ }^{5}$ with estimates of CKD prevalence ranging from $2 \%$ in Cote d'Ivoire to $30 \%$ in Zimbabwe. ${ }^{5}$ However, given the heterogeneity of the CKD definition (based on different equations to estimate glomerular filtration rate (GFR)) used in these studies, these figures could be an inaccurate estimation. Certainly, a number of these studies used the Cockcroft-Gault equation, which may not be suitable for use among individuals of black ancestry, and some of these studies did not include a nationally representative population-based sample. Therefore, in many LMICs or regions within these countries, data on the burden of CKD are still lacking, mainly due to an absence of quality resources and inadequate data collection methods. ${ }^{56}$

The major CKD risk factors include T2D, hypertension, obesity, ageing and black ethnicity. ${ }^{10}{ }^{17}$ LMICs, in particular those in Africa, are experiencing an accelerated increase in non-infectious disease, mainly hypertension and T2D, which contributed to $>15 \%$ of CKD cases. $^{18}$ Furthermore, infectious diseases, such as HIV, schistosomiasis and leishmaniasis, which also contribute to CKD, are highly prevalent in LMICs. ${ }^{11}$ In sub-Saharan Africa more than 22 million individuals have HIV, accounting for more than $70 \%$ of the global burden of infection, ${ }^{19}$ with HIV-associated nephropathy (HIVAN) fast becoming an important cause of morbidity and mortality related to kidney disease. ${ }^{12}{ }^{20}$ Moreover, individuals of African descent have a higher prevalence of CKD compared with individuals from European descent, suggesting a possible genetic predisposition in African populations. $^{21}{ }^{22}$ Apolipoprotein L1 (APOL1), which is fairly common throughout Africa, ${ }^{23}$ accounts for much of the excess genetic risk of CKD among individuals of African ancestry. ${ }^{811212224}$ This is particularly evident in 
individuals with two risk alleles, ${ }^{11}{ }^{23}$ as they experience a more rapid progression to ESRD. ${ }^{21}$

In LMICs, awareness of CKD remains low, ${ }^{25-27}$ particularly during the early asymptomatic stages of the disease. Jha $e t a l^{2}$ estimated that only one-third of people with CKD are diagnosed. These findings are corroborated by other studies also reporting very low rates of detection, such as in Northern Tanzania, where in a sample of 606 participants from 431 urban and rural households, only $10 \%$ of individuals with CKD were aware of their condition. ${ }^{6}$ Similarly, 23\% of an adult population living in Northern Senegal, $3.5 \%$ in $\operatorname{Taiwan}^{28}$ and $9.7 \%$ in China $^{29}$ were aware of their disease before intervention. ${ }^{30}$ In a more recent cross-sectional study in LMICs, it was documented that $6 \%$ of the general population and about $10 \%$ of high-risk individuals were aware they had CKD. ${ }^{16}$ These low rates of CKD awareness result in a large number of people progressing from CKD to ESRD, ${ }^{14}{ }^{27}$ which is in most cases fatal, particularly in LMICs where treatment is unavailable and unaffordable. According to the Global Burden of Disease (GBD) Study, 956200 deaths worldwide were directly attributable to CKD in 2013, mainly due to lack of access to treatment. ${ }^{31}$ Currently, only 20 per million population (pmp) and $68 \mathrm{pmp}$ in low-income countries and LMICs, respectively, receive dialysis treatment, versus $273 \mathrm{pmp}$ and $1064 \mathrm{pmp}$ in upper-middle-income to high-income countries. ${ }^{13}$

The economic burden of CKD has seldom been evaluated in LMICs; however, it is estimated to be significantly higher than observed in developed countries. ${ }^{2}$ In LMICs, the majority of patients present to hospital at late stages of CKD (ESRD), when dialysis is required, ${ }^{32}$ adding to treatment costs. Most people in LMICs have no health insurance and limited access to healthcare, which makes treatment for ESRD unaffordable and diagnosis of ESRD essentially a death sentence. ${ }^{43} 34$ In settings where limited renal care is available, patients with ESRD seldom have access to RRT, due to the high cost. ${ }^{35}{ }^{36}$ Globally, only about $7 \%$ of patients receiving RRT in 2010 were from LMICs. ${ }^{14}$ In India and Pakistan, for example, only about $5 \%$ of all patients with ESRD receive RRT. ${ }^{37}$ In North Africa (Egypt, Libya, Tunisia, Algeria, Morocco), less than $5 \%$ of patients with ESRD receive renal transplantation. ${ }^{38}$ It has been estimated that one session of dialysis costs US $\$ 100$ in Nigeria ${ }^{10}$ and between US $\$ 20$ and US\$60 in India. ${ }^{33}$ In China, the annual cost of dialysis is estimated at US $\$ 14300$ per patient, ${ }^{39}$ and in Africa the estimated annual cost of dialysis ranges between US $\$ 8000$ and US $\$ 12000$ per patient. ${ }^{10}$

Some governments have introduced the subsidisation of treatment, by instituting insurance schemes for the poor. For instance, in Nigeria, after several years of discussion and dialogue through the local nephrology association, the federal government has recently agreed to provide cover for the first six sessions of dialysis, with the possibility of extension to the first 3 months. ${ }^{40}$ The Chinese government has also implemented insurance schemes for the poor; however, patients in rural areas are still responsible for paying $35 \%-45 \%$ of the total cost, which is unaffordable for most. ${ }^{39}$ In India, certain states have started schemes to provide free RRT to the poor; however, coverage is limited. ${ }^{41}$ Although this is not nearly enough, it is a positive step in the right direction that will benefit patients and their families with access to RRT. Overall, given the high prevalence of CKD and the high costs of treatment during the advanced stages of the disease, the accessibility to treatment in LMICs remains low compared with developed countries. Whether the various schemes adopted by governments to facilitate access to RRT would be effectively implemented and translate into outcomes improvement remains to be established.

\section{Natural history of CKD among populations in LMICs}

The natural history of CKD is relatively well understood. CKD, which is defined as a reduced GFR $(<60 \mathrm{~mL} /$ $\mathrm{min} / 1.73 \mathrm{~m}^{2}$ for $\geq 3$ months) and/or increased urinary albumin excretion ( $\geq 30 \mathrm{mg} / 24$ hours), is classified into five stages, based on the estimated GFR (eGFR). Stages 1-3 are considered the early stages of CKD and nearly always asymptomatic. ${ }^{42}$ Stages 4 and 5 are classified as ESRD and are characterised by multiple complications. ${ }^{42}$ Once CKD is established, the natural expectation is that of disease progression, with each subsequent stage representing a deterioration from the previous stage, until ESRD is reached and ultimately death in the absence of intervention. The pace of CKD progression is variable and is a function of the underlying aetiological factors, comorbidities and the management thereof, and other factors operating specifically in the milieu of established CKD. ${ }^{10}{ }^{17}$ The interval from onset of CKD stage 3 to kidney failure is around 12 years, ${ }^{43}$ but the course of CKD progression can be halted at any time due to mortality from competing risk, particularly cardiovascular diseases (CVD).

The GBD expert group estimated that in 2013, reduced GFR accounted for $4 \%$ of deaths worldwide, with over $50 \%$ of these related to CVD and the remaining to ESRD. ${ }^{44}$ In this study, mortality from CVD in people with reduced GFR was slightly higher in LMICs than in developed countries. ${ }^{44}$ Furthermore, the mortality rate declined by $9 \%$ from 1990 in developed countries, while a $44 \%$ decline occurred in developing countries within the same time window. ${ }^{44}$ The presence of CKD is independently associated with increased rates of CVD, and the risk of death due to CVD linearly increases with decreasing eGFRs below $75 \mathrm{~mL} / \mathrm{min} / 1.73 \mathrm{~m}^{2} .{ }^{45} 46$ Thus, a small proportion of patients actually reach the terminal phase of CKD, as most die as a result of CVD prior to reaching ESRD. Accordingly, the prevalence rate (expressed as number of patients per million population) of ESRD in LMICs is much less than that observed in developed countries. ${ }^{47}$ For example, in Japan more than 2000/million people have ESRD, 1500/million people in the USA and roughly 800/million in Europe. Conversely, in LMICs such as South Asia ESRD affects between 100/million people and 200/million people, and less than 100/million people in sub-Saharan Africa and India. ${ }^{48} 49$ 
In LMICs, various factors contribute to the high risk of progression to ESRD. ${ }^{142743}$ First, there is a lack of optimal blood pressure control in patients with hypertension, where more than $90 \%$ of hypertension cases are uncontrolled in these settings. ${ }^{5051}$ Furthermore, uncontrolled T2D is of great concern in LMICs, with diabetic nephropathy being the leading cause of ESRD and affecting one in three patients with diabetes. ${ }^{52} \mathrm{HIV} / \mathrm{AIDS}$ is also known to accelerate CKD progression and is very prevalent in LMICs. This is particularly concerning as nearly $70 \%$ of HIV-infected individuals are concentrated in LMICs, and of those infected only $38 \%$ are currently receiving antiretroviral therapy (ART). ${ }^{19}$ In the absence of effective ART, approximately $50 \%$ of patients with HIVAN progress to ESRD within 2 years. ${ }^{53}$ ART is known to decrease the risk of renal disease in patients with HIV by $46 \%$ compared with naïve patients; however, some ART treatments (such as tenofovir disoproxil fumarate, ritonavir-boosted atazanavir and ritonavir-boosted lopinavir) have been associated with renal impairment. ${ }^{545}$ The use of herbal medicines, which is common in rural populations in Africa and Asia, ${ }^{56-58}$ can also act as nephrotoxins, thus increasing the risk of CKD progression.

\section{WHICH SCREENING TESTS TO USE IN LMICS?}

The first line of screening tools could consist of risk scores and questionnaires to determine risk of CKD, which are followed by biochemical tests, comprising eGFR and albuminuria tests. These tests may be used alone or in combination for detecting the specific stages of the diseases. ${ }^{59}$ Based on their advantages and limitations, some of the screening tests may be suitable for use in LMIC setting.

\section{Risk scores and questionnaires}

Various estimation/prediction models have been developed to be used as screening methods for the prevalence of CKD and its progression. ${ }^{60}$ These risk models are potentially ideal tools for large-scale CKD risk screening; however, risk models tend to be population-specific. ${ }^{60}$ More so, the participants used to develop CKD prediction models are more often of European origin. This is problematic because a homogeneous population does not allow for accurate determination of variability in CKD risk, and black ethnicity is particularly prone to CKD.

A systematic review of CKD risk prediction tools found that the discriminative performance of these models is generally acceptable-to-good in the population in which it was developed ${ }^{60}$ and modest-to-acceptable if tested in another population. ${ }^{60-62}$ However, only a few of the existing CKD risk algorithms have been validated in different populations and seldom in populations of LMICs. ${ }^{63-67}$ Among all the models, the cross-sectional population-based survey Screening for Occult Renal Disease (SCORED) appears to be a fairly reliable model for CKD risk prediction, as it has been validated various times, in mixed ethnic groups and with reasonable discrimination. ${ }^{63} 68$ However, in resource-poor settings, a simple prediction model that does not incorporate laboratory-based predictors is essential as it will keep costs low and reduce the screening burden. Therefore, a possible drawback to the use of the SCORED model is the cost attached to determining the presence of anaemia and T2D. To diagnose anaemia, a full blood count is needed, and to more accurately diagnose T2D particularly in black populations a 2-hour glucose tolerance test is advocated. ${ }^{69}$ Even so, the SCORED model is a possible candidate to be used in LMICs; however, the model needs to be validated and calibrated for populations in these settings. ${ }^{6370} 71$

\section{Biochemical tests}

Currently the key markers used to detect CKD include a persistent reduction in the eGFR $\left(<60 \mathrm{~mL} / \mathrm{min} / 1.73 \mathrm{~m}^{2}\right.$ for $\geq 3$ months) and abnormal urine albumin levels ( $\geq 30 \mathrm{mg} /$ day). ${ }^{1}$ Several biochemical screening tests, with various practical advantages and limitations, have been evaluated for CKD screening and can potentially be used in LMICs.

\section{Glomerular filtration rate}

GFR is measured indirectly by assessing the rate of clearance of markers such as inulin, iothalamate, iohexol, ethylenediaminetetraacetic acid or diethylenetriaminepentaacetic acid. ${ }^{72}$ However, these methods are expensive, cumbersome and time-consuming and not useful in general population screening or in routine clinical practice.

Therefore, the eGFR using prediction equations has been adopted as a routine measure of renal function. The most popular equations use serum creatinine level, although cystatin C-based equations have also been developed to estimate kidney function ${ }^{73}$ and have been reported to better predict all-cause mortality and cardiovascular events in older people, and all-cause mortality and ESRD in the general adult population. ${ }^{75}$ However, in LMICs the use of creatinine would be favoured above the use of cystatin C, as serum creatinine determination is a simple and less expensive and it is an internationally standardised test. ${ }^{76}$

The three commonly used creatinine-based estimators of GFR for adults include the Cockcroft-Gault, ${ }^{77}$ the Modification of Diet in Renal Disease (MDRD) ${ }^{78}$ and the CKD Epidemiology Collaboration (CKD-EPI) equations. ${ }^{79}$ The latter two equations contain a correction factor for ethnicity. However, the addition of a correction factor for ethnicity may result in misdiagnosis of CKD and is not a valid approach in all settings. ${ }^{8081}$ Various studies have compared the prevalence of CKD based on the different eGFR equations, ${ }^{82-87}$ and results show that CKD prevalence differs depending on which equation is used and on which population the equation is based. ${ }^{8081}$ For example, in studies conducted in populations in sub-Saharan Africa ${ }^{882}$ and predominant Caucasian populations, ${ }^{86}$ the MDRD equation had an advantage over the CKD-EPI equation. These studies are 
however limited by the fact that the reference standard for estimating GFR was not used. Conversely, studies conducted in Asian populations favour the use of the CKD-EPI equation above MDRD equation. ${ }^{83} 8487$ Even though these equations have not been validated for use in LMICs, there is a need to include eGFR (either MDRD or CKD-EPI) in routine practice, as this would give an indication for the presence of kidney disease and could lead to early referral and intervention. ${ }^{88}$

\section{Albuminuria}

Albuminuria is also a frequently used index of kidney function. ${ }^{72}$ The 24-hour urinary albumin concentration (UAC) is considered to be the reference standard. This method entails urine collection commencing immediately after discarding first morning urine and collecting all urine in the same container (including final voided urine next morning) until completion of 24 hours. ${ }^{89}$ This method is time-consuming and cumbersome as sample collection errors may occur and patient compliance is usually low. To avoid these limitations, random urine samples can be used for quantification of albuminuria, by calculating the urinary albumin to creatinine ratio (ACR).$^{72}$ Here, midstream urines are collected for a random urine sample. ${ }^{89}$ It is important that during the 24 hours and spot urine collection period, no heavy muscular work should be done as this will influence the serum creatinine concentration. The ACR has been found to correlate well with 24-hour UAC, ${ }^{89-91}$ with a high sensitivity (ranging between $79 \%$ and $87.6 \%$ ) and specificity (ranging between $87.5 \%$ and $95.5 \%$ ) to detect microalbuminuria.

\section{WHICH THERAPIES ARE AVAILABLE FOR SCREEN-DETECTED PATIENTS, AND HOW CAN THESE BE IMPLEMENTED IN LMIC SETTINGS?}

Interventions, if implemented timely, can slow the progression of CKD and subsequently reduce the risk of death due to CVD or ESRD. ${ }^{2}$ During CKD stages 1 and 2 , the general focus should be on aggressively treating the comorbid conditions, including elevated blood pressure and glucose levels and infectious diseases. The Joint National Committee for the Prevention, Detection, Evaluation, and Treatment of High Blood Pressure recommends a lower blood pressure goal for patients with decreased kidney function $(<130 / 85 \mathrm{~mm} \mathrm{Hg}$ if urine protein excretion is $<1 \mathrm{~g} /$ day, and $<125 / 75 \mathrm{~mm} \mathrm{Hg}$ if urine protein excretion is $>1 \mathrm{~g}$ /day) ${ }^{92}$ Various studies have shown that antihypertensive regimens containing ACE inhibitors or angiotensin receptor blockers are effective in delaying the progression of CKD, independent of blood pressure effects, ${ }^{93-97}$ by a sixth for every $5 \mathrm{~mm} \mathrm{Hg}$ reduction. ${ }^{98}$ Intensive blood pressure lowering has also been shown to reduce the relative risk of ESRD by $11 \%$ and produce a $10 \%$ reduction in the risk of albuminuria. ${ }^{99}$ Large clinical trials have also provided strong evidence that intensive glycaemic control (haemoglobin A1c target $\leq 6.5 \%$ ) in patients with diabetes reduces the risk of ESRD by $65 \%$, and reduces the relative risk of developing microalbuminuria by $9 \%^{100}$ and macroalbuminuria by $33 \% .^{101} 102$ CKD is a frequent complication of HIV infection and the use of ART is known to decrease the risk of CKD progression; however, meta-analytic results showed a significantly increased CKD risk in patients treated with tenofovir disoproxil fumarate-containing ARTs as compared with those receiving other regimens. ${ }^{54}$ Thus, the choice of ART is an extremely important decision in terms of managing HIV-infected patients, particularly those at high risk of CKD.

During stage 3 of CKD, in addition to continuing with the measures described above, the focus should shift to evaluating and treating the complications of CKD. During the advanced stages of CKD, patients are particularly at risk for developing anaemia, which occurs in about $90 \%$ of patients receiving RRT, ${ }^{103}$ the predominant cause being failure of the kidneys to produce enough endogenous erythropoietin (EPO), accompanying the fall in GFR. Even though EPO is the standard treatment of anaemia in CKD and associated with improved CVD outcome, ${ }^{104}$ the use thereof is limited in LMICs, due to the cost associated with this treatment. ${ }^{104-107}$ Bone and mineral metabolism is also compromised during CKD, with patients having an exceptionally high rate of severe vitamin $\mathrm{D}$ deficiency, which is further exacerbated by the reduced ability to convert $25-(\mathrm{OH})$ vitamin $\mathrm{D}$ into the active form, 1,25 di-hydroxy vitamin D. ${ }^{108}$ Vitamin D deficiency results in low calcium levels and hyperparathyroidism, a common complication in patients with CKD. Use of vitamin D and calcium supplements in these instances is feasible in LMICs. In addition to the decline in kidney function, the kidney becomes less efficient in excreting excess phosphate, resulting in phosphate build-up, ${ }^{108}$ a condition that can also be addressed irrespective of the setting.

By stage 4, referral to a nephrologist for RRT preparation, including dialysis, transplantation or both, should start. The death rate at the early stage of starting RRT in LMICs is very high, due essentially to the fact that most people with ERSD are referred at a very late stage and never get prepared on time for the start of long-term dialysis. ${ }^{109110}$ Early referral to a nephrologist has been found to decrease the chance of developing fatal complications associated with CKD substantially.111

\section{HOW FEASIBLE, EFFECTIVE AND COST-EFFECTIVE WILL SCREENING BE FOR CKD IN LMICS?}

A number of CKD screening programmes have been implemented in LMICs, of which the programme set up by the BP Koirala Institute of Health Science in Dharan, Nepal, in collaboration with International Society of Nephrology (ISN) and the Clinical Research Center of the Mario Negri Institute in Bergamo, Italy, is an example. ${ }^{112}$ In this study, 20811 individuals aged $>18$ years were screened, and data on family and personal medical history of disease (CKD, hypertension, T2D and 
CVD), treatments received for these conditions, demographic data, diet, smoking habit, alcohol consumption, physical activity, height, weight and blood pressure were collected. A spot urine protein, fasting blood glucose and serum creatinine concentrations were also assessed. Of the individuals screened, 4471 individuals had high blood pressure, high fasting glucose, proteinuria and impaired renal function. These high-risk individuals were followed up annually for 3 years and prescribed low-cost antihypertensives, antidiabetic or renoprotective medication, depending on the need. After the 3-year follow-up, $63 \%$ of the individuals with proteinuria at baseline had normal values and $48 \%$ of individuals with mild-to-moderate renal insufficiency at baseline had stable renal function. ${ }^{112}$ This study shows the feasibility of a screening and management programme in a low-resource setting, with a potential for population-wide benefit to managing CKD.

Often, a strong argument against screening or early detection is the cost-effectiveness of such an undertaking. ${ }^{113}$ Implementing screening for CKD in resource-poor settings can be very challenging logistically, time-consuming and expensive. However, whether the cost of screening followed by treatment outweighs the huge burden of this disease on the individual and the health system is still unknown. More economic research is needed in LMICs in order for health policymakers to prioritise CKD screening. Once policy is in place, an effective screening system can be implemented; however, the healthcare system would need to redirect existing resources as country budgets are most often limited. Cost-effectiveness of CKD screening needs to be assessed and evaluated against other health interventions. Studies from developed countries have demonstrated the feasibility of early detection and treatment of CKD. ${ }^{2}$ In particular, these studies showed that screening for CKD would be beneficial and cost-effective, when screening is geared more towards high-risk groups (hypertension, T2D and the elderly) compared with that of the general population, and screening followed by lifestyle modifications or treatment offers better cost-effective ratios. ${ }^{1459} 114$

Currently, there are limited data on the cost-effectiveness of CKD screening in LMICs. In a systematic review of 10 studies (8 studies of proteinuria and 2 studies of eGFR), the cost-effectiveness ratios for proteinuria ranged from \$14063-\$160 018/quality-adjusted life years (QALY) in the general population, \$5298-\$54 943/QALY in the diabetic population and \$23028-\$73 939/QALY in the hypertensive population. ${ }^{113}$ Additionally, the cost-effectiveness ratios for eGFR screening were $\$ 23680$ /QALY in the diabetic population and \$100253-\$109 912/QALY in the general population. For both eGFR and proteinuria, the cost of screening for CKD in the general population was higher than screening only the high-risk populations, which include those individuals with known hypertension and T2D. Another study found that compared with no screening, screening for CKD in the general population was associated with a cost of $\$$ C104 900/QALY. ${ }^{115}$ In subgroups of people with T2D and hypertension, the cost per QALY gained was \$C22 600 and \$C334 000, respectively. ${ }^{15}$ The current data on cost-effectiveness models are however from developed countries, which have a much higher per-person gross domestic product (GDP) and thus cost-effectiveness threshold. According to the WHO Commission of Macroeconomics and Health, the cost-effectiveness of an intervention depends on the local GDP. ${ }^{2}$ Therefore cost-effectiveness studies should be done in all regions.

\section{WHICH SCREENING STRATEGY TO ADOPT IN LMICS?}

Screening may be population-based or conducted in the context of healthcare (opportunistic screening). Population-based screening attempts to screen the entire population at risk, and opportunistic screening involves screening individuals as part of a routine medical visit for other purposes. ${ }^{116}$ Both these screening methods have merit, but neither meets the screening needs in all circumstances.

Screening high-risk individuals for CKD, such as those with hypertension, T2D, HIV infection and $>60$ years of age, is already included in clinical practice guidelines in several countries around the world, ${ }^{117-119}$ and would be a viable option when mass campaigns are not possible or resources are limited. However, in LMICs a very large proportion of patients at high risk remain undiagnosed, ${ }^{16} 50120$ mainly because they do not have access to healthcare. A recent systematic review showed that only $18 \%$ of the adult African population with hypertension were aware of their condition. ${ }^{50}$ However, even among populations already in regular contact with healthcare systems, hypertension remains unrecognised, with $70 \%$ of patients with HIV ${ }^{121}$ and more than $10 \%$ of patients with diabetes in Africa not being aware of their comorbid hypertension. ${ }^{122}$

However, the use of the presence of major risk factors as a starting point for CKD screening in clinical settings has relevance for a number of purposes: (1) The yield of screening is likely to be high and for low investment considering the high prevalence or incidence of CKD in the presence of those risk factors; (2) treatment routinely used to control those factors on their own can in turn prevent the onset or slow the progression of CKD; and (3) the presence of CKD can however invite the intensification of treatments targeting those risk factors, to maximise their beneficial effects on CKD prevention and/or the modification of those treatments to introduce compounds that are safer for the kidney or have better renal protection properties. In the absence of strategies to improve the detection of those risk factors, targeting only populations with levels defining high-risk status for CKD screening will have a limited impact on the burden of undiagnosed CKD in general in LMICs. A large proportion of people with CKD is likely made up of people with a combination of non-optimal levels of risk factors, but below the threshold for defining disease states (such as prediabetes or prehypertension), and therefore not targeted for CKD screening in routine practice. 
Screening for CKD in high-risk individuals would be based on eGFR and urinary albumin excretion. This may not be widely achievable in primary care setting particularly the underserved rural ones in LMICs, where laboratory investigations may not always be routinely available. The approach will also become expensive in routine care setting when considering expanding CKD screening to those with a combination of non-optimal level of risk factors. Parameters included in prevalent CKD risk models, not requiring laboratory tests, such as history of diseases and treatment, blood pressure level, age, gender and others, are routinely collected in clinical settings in LMICs. Therefore, in addition to targeting high-risk individuals for CKD screening (using eGFR and/or urinary albumin excretion where available), validated CKD risk models could be used in routine practice in LMICs. This could aid in identifying individuals at high risk in settings where laboratory investigations are not available. These individuals can then be referred or prescribed laboratory test for CKD screening. Furthermore, among those eventually diagnosed with CKD, validated CKD progression models can be used to identify those patients who are more likely to progress to ESRD, and thus need interventions to slow CKD progression. Risk prediction models are (or can be made) available in formats easily applicable (smartphone versions for instance) in routine settings with little extra effort, facilitated by lay health workers.

Across LMICs, there is a lack of access to healthcare services where opportunistic screening for CKD and risk factors can occur. In South Africa for instance, the country with the most developed healthcare system in sub-Saharan Africa, less than $20 \%$ of the population has continuous contact with the health system. Therefore, using only hospital-based strategies is unlikely to achieve significant reduction in the burden of undiagnosed CKD, necessitating further strategies to identify those beyond the traditional healthcare setting.

Lessons learnt from preventing and controlling infectious diseases suggest that community-based outreach activities play a major role in improving access to health interventions. Access to immunisation has been achieved in Africa for instance, among others, through community-based vaccination campaigns by nurses and community health workers. Interestingly, some of these community-based activities have been successfully adapted for chronic infectious conditions like HIV/AIDS and tuberculosis. There is therefore already an opportunity to implement community-based CKD screening as a by-product of existing programmes for chronic infectious diseases, or to use such programmes to inform those of CKD. For community-based screening of CKD to be attractive and useful, it should not be considered in isolation, but rather in the context of coscreening for major risk factors such as hypertension and T2D. The availability of affordable electronic blood pressure measuring devices and point-of-care glucose monitors makes community-based screening for those factors achievable. A simple and practical strategy could consist of using community-based hypertension status assessment as an entry point, followed by the implementation of non-laboratory-based risk scores to identify high-risk individuals for CKD and T2D. Those with prevalent hypertension, T2D and/or high risk of CKD or T2D then get referred to the healthcare facilities for confirmation and further screening via laboratory tests.

The course of action following screening is likely setting-specific. In health facility-based settings, those diagnosed with a CKD should benefit from additional screening to identify modifiable risk and aetiological factors, and receive optimal risk factor control and renoprotective interventions to prevent/slow the progression of the disease, bearing in mind that these interventions could be less accessible or affordable in some settings. Patients diagnosed with advanced/progressing (in spite of appropriate interventions) CKD should be referred to nephrologists or relevant specialists where additional aetiological investigations and refined treatments could be implemented, or timely preparation started for patients who would ultimately need RRT. Ideally, community-based screening should occur in the context of health promotion activities targeting reductions in the overall population risk of CKD and major risk factors.

While the limited coping capacity has been cited as a deterrent to $\mathrm{CKD}$ and non-communicable diseases (NCD) screening in LMICs, there are possible strategies to overcome some of these limitations. Risk screening for CKD and NCDs at large can be implemented both in health facilities and at community level using trained lay health workers, simplified clinical pathways and taking advantages of existing technologies. With little additional training, low-level health workers can efficiently prescribe and tailor most treatments needed to mitigate CKD risk. ${ }^{123}$ Their availability also increases the prospect of implementing community-based high-risk approach to CVD (and by extension CKD) risk reduction, using for instance trained community health workers and simplified algorithms.

\section{CONCLUSION}

Extant evidence suggests that CKD screening should receive attention in LMICs. CKD is increasingly common in these settings, due to the significant increase in non-infectious disease (particularly T2D and hypertension), as well as infectious conditions such as HIV. To decrease the high mortality and morbidity associated with CKD, early detection is essential because therapeutic interventions are likely to be effective if implemented early in the course of the disease. Additional research is needed on screening programmes in LMICs as most available studies were conducted in developed countries. It should however be emphasised that the implementation of these interventions in real-world settings of LMICs can pose a challenge to the already burdened health systems, which currently deliver suboptimal care. Furthermore, cost-effectiveness of screening for CKD should be investigated 
extensively in LMICs, as one cannot infer cost-effectiveness across countries. Designing policies for CKD screening offers a unique opportunity to scale up CKD care and by so doing strengthen the healthcare system of LMICs, and thus potentially limiting future healthcare costs.

Correction notice This paper has been amended since it was published Online First. Owing to a scripting error, some of the publisher names in the references were replaced with 'BMJ Publishing Group'. This only affected the full text version, not the PDF. We have since corrected these errors and the correct publishers have been inserted into the references.

Contributors CG, JBE and APK contributed to conception and design of the manuscript. CG, AM, I0, JBE and APK were involved in drafting the manuscript. All authors critically revised the manuscript for important intellectual content and approved the final version of the manuscript.

Funding Financial support for this study was provided by theSouth African Medical Research Council

Competing interests All authors have completed the ICMJE uniform disclosure form at www.icmje.org/coi_disclosure.pdf and declare no support from any organisation for the submitted work, no financial relationships with any organisations that might have an interest in the submitted work in the previous 3 years, and no other relationships or activities that could appear to have influenced the submitted work.

Provenance and peer review Not commissioned; externally peer reviewed.

Open Access This is an Open Access article distributed in accordance with the Creative Commons Attribution Non Commercial (CC BY-NC 4.0) license, which permits others to distribute, remix, adapt, build upon this work non-commercially, and license their derivative works on different terms, provided the original work is properly cited and the use is non-commercial. See: http://creativecommons.org/ licenses/by-nc/4.0/

(c) Article author(s) (or their employer(s) unless otherwise stated in the text of the article) 2017. All rights reserved. No commercial use is permitted unless otherwise expressly granted.

\section{REFERENCES}

1. Bolton $\mathrm{K}$, Culleton B, Harvey K. K/DOQI clinical practice guidelines for chronic kidney disease: evaluation, classification, and stratification kidney disease outcome quality initiative. Am JKidney Dis 2002;39(2 Suppl 1):S1-246.

2. Jha V, Garcia-Garcia G, Iseki K, et al. Chronic kidney disease: global dimension and perspectives. Lancet 2013;382:260-72.

3. Mills KT, Xu Y, Zhang W, et al. A systematic analysis of worldwide population-based data on the global burden of chronic kidney disease in 2010. Kidney Int 2015;88:950-7.

4. Lozano R, Naghavi M, Foreman K, et al. Global and regional mortality from 235 causes of death for 20 age groups in 1990 and 2010: a systematic analysis for the global burden of disease study 2010. Lancet 2012;380:2095-128.

5. Stanifer JW, Jing B, Tolan S, et al. The epidemiology of chronic kidney disease in sub-Saharan Africa: a systematic review and meta-analysis. Lancet Glob Health 2014;2:e174-e181.

6. Stanifer JW, Muiru A, Jafar TH, et al. Chronic kidney disease in low- and middle-income countries. Nephrol Dial Transplant 2016;31:868-74.

7. Arogundade FA, Barsoum RS. CKD prevention in Sub-Saharan Africa: a call for governmental, nongovernmental, and community support. Am J Kidney Dis 2008;51:515-23.

8. Matsha TE, Yako YY, Rensburg MA, et al. Chronic kidney diseases in mixed ancestry South African populations: prevalence, determinants and concordance between kidney function estimators. BMC Nephrol 2013;14:75.

9. Meguid El Nahas A, Bello AK. Chronic kidney disease: the global challenge. Lancet 2005;365:331-40.

10. Ayodele OE, Alebiosu CO. Burden of chronic kidney disease: an international perspective. Adv Chronic Kidney Dis 2010;17:215-24.

11. Kasembeli AN, Duarte R, Ramsay M, et al. APOL1 risk variants are strongly associated with HIV-Associated nephropathy in black South Africans. J Am Soc Nephrol 2015;26:2882-90.

12. Wearne N, Okpechi IG. HIV-associated renal disease - an overview. Clin Nephrol 2016;86:41-7.
13. Liyanage $\mathrm{T}$, Ninomiya $\mathrm{T}$, Jha $\mathrm{V}$, et al. Worldwide access to treatment for end-stage kidney disease: a systematic review. Lancet 2015;385:1975-82.

14. Garcia-Garcia G, Jha V. World Kidney Day Steering C. CKD in disadvantaged populations. Kidney Int 2015;87:251-3.

15. Wilson JM, Jungner YG. [Principles and practice of mass screening for disease]. Bol Oficina Sanit Panam 1968;65:281-393.

16. Ene-lordache B, Perico N, Bikbov B, et al. Chronic kidney disease and cardiovascular risk in six regions of the world (ISN-KDDC): a cross-sectional study. Lancet Glob Health 2016;4:e307-e319.

17. Agyei-Mensah S, de-Graft Aikins A. Epidemiological transition and the double burden of disease in Accra, Ghana. J Urban Health 2010;87:879-97.

18. Katz IJ, Gerntholtz TE, van Deventer M, et al. Is there a need for early detection programs for chronic kidney disease? Clin Nephrol 2010;74(Suppl 1):S113-8.

19. Wang H, Wolock TM, Carter A, et al. Estimates of global, regional, and national incidence, prevalence, and mortality of HIV, 19802015: the global burden of disease study 2015. Lancet HIV 2016;3:e361-87.

20. Han TM, Naicker S, Ramdial PK, et al. A cross-sectional study of HIV-seropositive patients with varying degrees of proteinuria in South Africa. Kidney Int 2006;69:2243-50.

21. Limou S, Nelson GW, Kopp JB, et al. APOL1 kidney risk alleles: population genetics and disease associations. Adv Chronic Kidney Dis 2014;21:426-33.

22. Parsa A, Kao WH, Xie D, et al. APOL1 risk variants, race, and progression of chronic kidney disease. N Engl J Med 2013;369:2183-96

23. Ulasi II, Tzur S, Wasser WG, et al. High population frequencies of APOL1 risk variants are associated with increased prevalence of non-diabetic chronic kidney disease in the igbo people from southeastern Nigeria. Nephron Clin Pract 2013;123(1-2):123-8.

24. Freedman BI. APOL1 and nephropathy progression in populations of African ancestry. Semin Nephrol 2013;33:425-32.

25. Coresh J, Byrd-Holt D, Astor BC, et al. Chronic kidney disease awareness, prevalence, and trends among U.S. adults, 1999 to 2000. J Am Soc Nephrol 2005;16:180-8.

26. Minutolo R, De Nicola L, Mazzaglia G, et al. Detection and awareness of moderate to advanced CKD by primary care practitioners: a cross-sectional study from Italy. Am J Kidney Dis 2008;52:444-53.

27. Perico N, Remuzzi G. Prevention programs for chronic kidney disease in low-income countries. Intern Emerg Med 2016;11:385-9.

28. Hwang SJ, Tsai JC, Chen HC. Epidemiology, impact and preventive care of chronic kidney disease in Taiwan. Nephrology 2010;15(Suppl 2):3-9.

29. Lu C, Zhao H, Xu G, et al. Prevalence and risk factors associated with chronic kidney disease in a Uygur adult population from Urumqi. J Huazhong Univ Sci Technolog Med Sci 2010;30:604-10.

30. Seck SM, Doupa D, Guéye L, et al. Epidemiology of chronic kidney disease in northern region of Senegal: a community-based study in 2012. Pan Afr Med J 2014;18:307.

31. Global Burden of Disease Study 2013 Collaborators. Global, regional, and national incidence, prevalence, and years lived with disability for 301 acute and chronic diseases and injuries in 188 countries, 1990-2013: a systematic analysis for the global burden of disease study 2013. Lancet 2015;386:743-800.

32. Parameswaran S, Geda SB, Rathi M, et al. Referral pattern of patients with end-stage renal disease at a public sector hospital and its impact on outcome. Natl Med J India 2011:24:208-13.

33. Chugh KS, Jha V, Chugh S. Economics of dialysis and renal transplantation in the developing world. Transplant Proc 1999:31:3275-7.

34. Hamer RA, EI Nahas AM. The burden of chronic kidney disease. BMJ 2006;332:563-4.

35. Antwi S. State of renal replacement therapy services in Ghana. Blood Purif 2015;39(1-3):137-40.

36. Moosa MR, Kidd M. The dangers of rationing dialysis treatment: the dilemma facing a developing country. Kidney Int 2006;70:1107-14.

37. Sakhuja V, Sud K. End-stage renal disease in India and Pakistan: burden of disease and management issues. Kidney Int Suppl 2003;83:S115-S118.

38. Barsoum RS. Burden of chronic kidney disease: North Africa. Kidney Int Supp/ 2013;3:164-6.

39. Zhang L, Wang H. Chronic kidney disease epidemic: cost and health care implications in China. Semin Nephrol 2009;29:483-6.

40. Bamgboye EL. The challenges of ESRD care in developing economies: sub-Saharan African opportunities for significant improvement. Clin Nephrol 2016;86:18-22. 
41. Jha V. Current status of chronic kidney disease care in Southeast Asia. Semin Nephrol 2009;29:487-96.

42. Kdigo. K. Clinical practice guideline for the evaluation and management of chronic kidney disease. Kidney Int 2012;3.

43. Levey AS, Coresh J. Chronic kidney disease. Lancet 2012;379:165-80.

44. Thomas B, Matsushita K, Abate KH, et al. Global cardiovascular and renal outcomes of reduced GFR. J Am Soc Nephrol 2017;28:ASN.2016050562.

45. Matsushita K, van der Velde M, et al. Association of estimated glomerular filtration rate and albuminuria with all-cause and cardiovascular mortality in general population cohorts: a collaborative meta-analysis. Lancet 2010;375:2073-81.

46. van der Velde M, Matsushita K, Coresh J, et al. Lower estimated glomerular filtration rate and higher albuminuria are associated with all-cause and cardiovascular mortality. A collaborative meta-analysis of high-risk population cohorts. Kidney Int 2011;79:1341-52.

47. Nugent RA, Fathima SF, Feigl AB, et al. The burden of chronic kidney disease on developing nations: a 21st century challenge in global health. Nephron Clin Pract 2011;118:c269-c277.

48. Agarwal SK, Srivastava RK. Chronic kidney disease in India: challenges and solutions. Nephron Clin Pract 2009;111:c197-c203.

49. Barsoum RS. Chronic kidney disease in the developing world. $N$ Engl J Med 2006;354:997-9.

50. Ataklte F, Erqou S, Kaptoge S, et al. Burden of undiagnosed hypertension in sub-saharan Africa: a systematic review and metaanalysis. Hypertension 2015;65:291-8.

51. Basu S, Millett C. Social epidemiology of hypertension in middleincome countries: determinants of prevalence, diagnosis, treatment, and control in the WHO SAGE study. Hypertension 2013;62:18-26.

52. Hossain MP, Goyder EC, Rigby JE, et al. CKD and poverty: a growing global challenge. Am J Kidney Dis 2009;53:166-74.

53. Hays T, Wyatt CM. APOL1 variants in HIV-associated nephropathy: just one piece of the puzzle. Kidney Int 2012;82:259-60.

54. Islam FM, Wu J, Jansson J, et al. Relative risk of renal disease among people living with HIV: a systematic review and metaanalysis. BMC Public Health 2012;12:234.

55. Mocroft A, Lundgren JD, Ross M, et al. Cumulative and current exposure to potentially nephrotoxic antiretrovirals and development of chronic kidney disease in HIV-positive individuals with a normal baseline estimated glomerular filtration rate: a prospective international cohort study. Lancet HIV 2016;3:e23-e32.

56. Jha V, Rathi M. Natural medicines causing acute kidney injury. Semin Nephrol 2008;28:416-28.

57. Guh JY, Chen HC, Tsai JF, et al. Herbal therapy is associated with the risk of CKD in adults not using analgesics in Taiwan. Am J Kidney Dis 2007;49:626-33.

58. Yang L, Su T, Li XM, et al. Aristolochic acid nephropathy: variation in presentation and prognosis. Nephrol Dial Transplant 2012;27:292-8.

59. Qaseem A, Hopkins RH, Sweet DE, et al. Screening, monitoring, and treatment of stage 1 to 3 chronic kidney disease: a clinical practice guideline from the American College of Physicians. Ann Intern Med 2013;159:835-47.

60. Echouffo-Tcheugui JB, Kengne AP. Risk models to predict chronic kidney disease and its progression: a systematic review. PLoS Med 2012;9:e1001344.

61. Mogueo A, Echouffo-Tcheugui JB, Matsha TE, et al. Validation of two prediction models of undiagnosed chronic kidney disease in mixed-ancestry South Africans. BMC Nephrol 2015;16:94.

62. Moons KG, Kengne AP, Grobbee DE, et al. Risk prediction models: II. External validation, model updating, and impact assessment. Heart 2012;98:691-8.

63. Bang H, Vupputuri S, Shoham DA, et al. SCreening for Occult REnal Disease (SCORED): a simple prediction model for chronic kidney disease. Arch Intern Med 2007;167:374-81.

64. Blech I, Katzenellenbogen M, Katzenellenbogen A, et al. Predicting diabetic nephropathy using a multifactorial genetic model. PLOS One 2011;6:e18743.

65. Chien KL, Lin HJ, Lee BC, et al. A prediction model for the risk of incident chronic kidney disease. Am J Med 2010;123:836-46.

66. O'Seaghdha CM, Lyass A, Massaro JM, et al. A risk score for chronic kidney disease in the general population. Am J Med 2012;125:270-7.

67. Tangri N, Stevens LA, Griffith J, et al. A predictive model for progression of chronic kidney disease to kidney failure. JAMA 2011;305:1553-9.

68. de Almeida EA, Lavinas C, Teixeira C, et al. Evaluation of an instrument for screening patients at risk for chronic kidney disease: testing SCORED (Screening for Occult Renal Disease) in a Portuguese population. Kidney Blood Press Res 2012;35:568-72.

69. Goedecke JH, Dave JA, Faulenbach MV, et al. Insulin response in relation to insulin sensitivity: an appropriate beta-cell response in black South African women. Diabetes Care 2009;32:860-5.

70. Bang H, Mazumdar M, Kern LM, et al. Validation and comparison of a novel screening guideline for kidney disease: KEEPing SCORED. Arch Intern Med 2008;168:432-5.

71. Bang H, Mazumdar M, Newman G, et al. Screening for kidney disease in vascular patients: SCreening for Occult REnal Disease (SCORED) experience. Nephrol Dial Transplant 2009;24:2452-7.

72. Vart P, Grams ME. Measuring and assessing kidney function. Semin Nephrol 2016;36:262-72.

73. Grubb A, Horio M, Hansson LO, et al. Generation of a new cystatin C-based estimating equation for glomerular filtration rate by use of 7 assays standardized to the international calibrator. Clin Chem 2014;60:974-86.

74. Inker LA, Schmid $\mathrm{CH}$, Tighiouart $\mathrm{H}$, et al. Estimating glomerular filtration rate from serum creatinine and cystatin C. N Engl J Med 2012;367:20-9.

75. Shlipak MG, Matsushita K, Ärnlöv J, et al. Cystatin C versus creatinine in determining risk based on kidney function. $N$ Engl $J$ Med 2013;369:932-43.

76. Myers GL, Miller WG, Coresh J, et al. Recommendations for improving serum creatinine measurement: a report from the laboratory working group of the national kidney disease education program. Clin Chem 2006;52:5-18.

77. Cockcroft DW, Gault MH. Prediction of creatinine clearance from serum creatinine. Nephron 1976;16:31-41.

78. Levey AS, Bosch JP, Lewis JB, et al. A more accurate method to estimate glomerular filtration rate from serum creatinine: a new prediction equation. Modification of Diet in Renal Disease Study Group. Ann Intern Med 1999;130:461-70.

79. Levey AS, Stevens LA, Schmid CH, et al. A new equation to estimate glomerular filtration rate. Ann Intern Med 2009;150:604-12

80. Eastwood JB, Kerry SM, Plange-Rhule J, et al. Assessment of GFR by four methods in adults in Ashanti, Ghana: the need for an eGFR equation for lean African populations. Nephrol Dial Transplant 2010;25:2178-87.

81. Madala ND, Nkwanyana N, Dubula T, et al. Predictive performance of eGFR equations in South Africans of African and Indian ancestry compared with ${ }^{99} \mathrm{mTc}-\mathrm{DTPA}$ imaging. Int Urol Nephrol 2012;44:847-55.

82. Agoons DD, Balti EV, Kaze FF, et al. Performance of three glomerular filtration rate estimation equations in a population of sub-Saharan Africans with type 2 diabetes. Diabet Med 2016;33:1291-8.

83. Horio M, Imai E, Yasuda $Y$, et al. Modification of the CKD epidemiology collaboration (CKD-EPI) equation for Japanese: accuracy and use for population estimates. Am J Kidney Dis 2010;56:32-8.

84. Hsu CY. CKD-EPI eGFR categories were better than MDRD categories for predicting mortality in a range of populations. Ann Intern Med 2012;157:JC5-12.

85. Mombelli CA, Giordani MC, Imperiali NC, et al. Comparison between CKD-EPI creatinine and MDRD equations to estimate glomerular filtration rate in kidney transplant patients. Transplant Proc 2016;48:625-30.

86. Ponte B, Pruijm M, Marques-Vidal P, et al. Determinants and burden of chronic kidney disease in the population-based CoLaus study: a cross-sectional analysis. Nephrol Dial Transplant 2013;28:2329-39

87. Teo BW, Xu H, Wang D, et al. GFR estimating equations in a multiethnic Asian population. Am J Kidney Dis 2011;58:56-63.

88. Johnson DW, Jones GR, Mathew TH, et al. Chronic kidney disease and automatic reporting of estimated glomerular filtration rate: new developments and revised recommendations. Med J Aust 2012;197:224-5.

89. Pathania M, Rathaur VK, Yadav N, et al. Quantitative microalbuminuria assessment from 'Random Voided Urinary Albumin: Creatinine Ratio' versus ' 24 hours Urinary Albumin Concentration' for screening of diabetic nephropathy. J Clin Diagn Res 2013;7:2828-31.

90. Gansevoort RT, Verhave JC, Hillege HL, et al. The validity of screening based on spot morning urine samples to detect subjects with microalbuminuria in the general population. Kidney Int Suppl 2005:94:S28-S35.

91. Methven S, MacGregor MS, Traynor JP, et al. Assessing proteinuria in chronic kidney disease: protein-creatinine ratio versus albumincreatinine ratio. Nephrol Dial Transplant 2010;25:2991-6. 
92. National High Blood Pressure Education P. The Seventh Report of the Joint National Committee on Prevention, detection, evaluation, and treatment of high blood pressure. Bethesda (MD): National Heart, Lung, and Blood Institute (US), 2004.

93. Düsing R. Mega clinical trials which have shaped the RAS intervention clinical practice. Ther Adv Cardiovasc Dis 2016:10:133-50.

94. Ferrari P. Prescribing angiotensin-converting enzyme inhibitors and angiotensin receptor blockers in chronic kidney disease. Nephrology 2007;12:81-9.

95. Komers R, Plotkin H. Dual inhibition of renin-angiotensinaldosterone system and endothelin-1 in treatment of chronic kidney disease. Am J Physiol Regul Integr Comp Physiol 2016;310:R87 7-R884.

96. Ripley E. Complementary effects of angiotensin-converting enzyme inhibitors and angiotensin receptor blockers in slowing the progression of chronic kidney disease. Am Heart J 2009;157(6 Suppl):S7-S16.

97. Sharma P, Blackburn RC, Parke CL, et al. Angiotensin-converting enzyme inhibitors and angiotensin receptor blockers for adults with early (stage 1 to 3) non-diabetic chronic kidney disease. Cochrane Database Syst Rev 2011;10:CD007751.

98. Ninomiya T, Perkovic V, Turnbull F, et al. Blood pressure lowering and major cardiovascular events in people with and without chronic kidney disease: meta-analysis of randomised controlled trials. BMJ 2013;347:f5680.

99. Lv J, Neal B, Ehteshami P, et al. Effects of intensive blood pressure lowering on cardiovascular and renal outcomes: a systematic review and meta-analysis. PLoS Med 2012;9:e1001293.

100. Jun M, Perkovic V, Cass A. Intensive glycemic control and renal outcome. Contrib Nephrol 2011;170:196-208.

101. Bilous R. Microvascular disease: what does the UKPDS tell us about diabetic nephropathy? Diabet Med 2008;25(Suppl 2):25-9.

102. Perkovic V, Heerspink HL, Chalmers J, et al. Intensive glucose control improves kidney outcomes in patients with type 2 diabetes. Kidney Int 2013;83:517-23.

103. Nakhoul G, Simon JF. Anemia of chronic kidney disease: Treat it, but not too aggressively. Cleve Clin J Med 2016;83:613-24.

104. Noce A, Dessì M, Durante O, et al. Influence of continuous erythropoietin receptor activator on markers of cardiovascular risk in chronic kidney disease patients. Int J Cardiol 2013;167:3070-2.

105. Adamu B, Ma'aii SM, Erwin PJ, et al. Meta-analysis of randomized controlled trials on androgens versus erythropoietin for anaemia of chronic kidney disease: implications for developing countries. Int $J$ Nephrol 2012;2012:1-6.

106. Clement FM, Klarenbach S, Tonelli M, et al. An economic evaluation of erythropoiesis-stimulating agents in CKD. Am J Kidney Dis 2010;56:1050-61.

107. Maïz HB, Abderrahim E, Zouaghi K. Anemia and end-stage renal disease in the developing world. Artif Organs 2002;26:760-4.
108. Williams ME. Chronic kidney disease/bone and mineral metabolism: the imperfect storm. Semin Nephrol 2009;29:97-104.

109. Halle MP, Takongue C, Kengne AP, et al. Epidemiological profile of patients with end stage renal disease in a referral hospital in Cameroon. BMC Nephrol 2015;16:59.

110. Kumar S, Jeganathan J, Amruthesh KN. Timing of nephrology referral: influence on mortality and morbidity in chronic kidney disease. Nephrourol Mon 2012;4:578-81.

111. Smart NA, Titus TT. Outcomes of early versus late nephrology referral in chronic kidney disease: a systematic review. Am J Med 2011;124:1073-80.

112. Sharma SK, Ghimire A, Carminati S, et al. Management of chronic kidney disease and its risk factors in eastern Nepal. Lancet Glob Health 2014;2:e506-e507.

113. Komenda P, Ferguson TW, Macdonald K, et al. Cost-effectiveness of primary screening for CKD: a systematic review. Am J Kidney Dis 2014;63:789-97.

114. Grootendorst DC, Jager KJ, Zoccali C, et al. Screening: why, when, and how. Kidney Int 2009;76:694-9.

115. Manns B, Hemmelgarn B, Tonelli M, et al. Population based screening for chronic kidney disease: cost effectiveness study. BMJ 2010;341:c5869.

116. Mathew T, Corso O. Review article: early detection of chronic kidney disease in Australia: which way to go? Nephrology 2009:14:367-73.

117. Crandall JP, Knowler WC, Kahn SE, et al. The prevention of type 2 diabetes. Nat Clin Pract Endocrinol Metab 2008;4:382-93.

118. Gillies CL, Abrams KR, Lambert PC, et al. Pharmacological and lifestyle interventions to prevent or delay type 2 diabetes in people with impaired glucose tolerance: systematic review and metaanalysis. BMJ 2007;334:299

119. Rosendaal NT, Hendriks ME, Verhagen MD, et al. Costs and cost-effectiveness of hypertension screening and treatment in adults with hypertension in Rural Nigeria in the context of a health insurance program. PLoS One 2016;11:e0157925.

120. Beagley J, Guariguata L, Weil C, et al. Global estimates of undiagnosed diabetes in adults. Diabetes Res Clin Pract 2014; 103:150-60

121. Mutemwa M, De Villiers A, Peer N, et al. ISH NIA PS 03-07 an assessment of the prevalence, detection, treatment and control of hypertension in HIV infected patients receiving care across public HIV care facilities in the western cape province of South Africa. $J$ Hypertens 2016;34:e282-e283.

122. Choukem SP, Kengne AP, Dehayem YM, et al. Hypertension in people with diabetes in Sub-Saharan Africa: revealing the hidden face of the iceberg. Diabetes Res Clin Pract 2007;77:293-9.

123. Joshi R, Alim M, Kengne AP, et al. Task shifting for noncommunicable disease management in low and middle income countries--a systematic review. PLoS One 2014;9:e103754. 